Scientific Paper

\title{
Hybrid Monte Carlo source model: Advantages and deficiencies
}

\author{
Déte VAN EEDEN ${ }^{1, a}$, Freek C.P. DU PLESSIS ${ }^{1}$ \\ ${ }^{1}$ Department of Medical Physics, University of the Free State, P O Box 339, Bloemfontein, 9300, South Africa \\ ${ }^{a}$ E-mail address: detevaneeden@gmail.com
}

(received 6 November 2017; accepted 13 February 2018)

\begin{abstract}
Monte Carlo (MC) simulation is the gold standard for dose calculation. An accurate mathematical source model can be used for the radiation beams. Source models can consist of sub-sources or fewer sources with data that need to be measured. This can speed up treatment plan verification without the need for a full simulation of the radiation treatment machine.

Aims: This study aimed to construct a novel hybrid source model for $6 \mathrm{MV}$ photon beams for an Elekta Synergy accelerator and to commission it against measured beam data and treatments plans.

Methods and Material: The model comprised of a circular photon and planar electron contamination source. The modified Schiff formula provided off-axis variable bremsstrahlung spectra. Collimation and scatter were modelled with error functions. An exponential function modelled the transmitted fluence through the collimators. The source model was commissioned by comparing simulated and measured MC data. Dose data included profiles, depth dose and film measurements in a Rando phantom. Field sizes ranged from $1 \times 1 \mathrm{~cm}^{2}$ to $40 \times 40 \mathrm{~cm}^{2}$.

Results: Regular, wedged and asymmetrical fields could be modelled within $1.5 \%$ or $1.5 \mathrm{~mm}$. More than $95 \%$ of all points lie within $3 \%$ or $3 \mathrm{~mm}$ for the multi-leaf collimators contours data. A gamma criterion of $3 \%$ or $3 \mathrm{~mm}$ was met for a complex treatment case.

Conclusions: The two sub-source model replicated clinical 6 MV Elekta Synergy photons beams and could calculate the dose accurately for conformal treatments in complex geometries such as a head-and-neck case.
\end{abstract}

Key words: hybrid source model; DOSXYZnrc; Monte Carlo; Elekta Synergy; particle source model.

\section{Introduction}

According to The Lancet, South Africa can expect a $78 \%$ increase in cancer cases by 2030. One in three people will develop cancer in their lifetime in the United Kingdom where the disease caused 430 mortalities per day in 2010 [1]. Radiation of tumours plays a prominent role and accurate radiation treatment planning is important for successful treatment outcomes.

Most radiation treatment planning systems (TPS) use analytical dose calculation algorithms, but Monte Carlo (MC) simulation of particle transport is the most accurate way to determine the absorbed dose in regions of tissue interfaces and heterogeneities, e.g. the lung and head-and-neck regions [1,27]. The DOSXYZnrc MC code is considered the gold standard to simulate dose deposition in computed tomography (CT)based patient models [8]. It can independently verify TPS dose calculations, albeit fast routine dose verification is required.

One of the difficulties with the clinical implementation of MC dose calculation is the characterisation of the radiation source within a universal source model.
A full MC simulation that includes the radiation treatment head of the machine would not be time efficient; any radiation field must first be completely simulated before its phase space data can be used in subsequent BEAM/DOSXYZnrc simulations. Phase space data contain the dynamical parameters of all particles followed through the simulation process ending up at a pre-determined scoring plane. The millions of particle histories in a phase space files can take up large amounts of disk space. Sometimes the exact geometry of the head is unknown and an alternative is to use a source model that describes the energy and fluence distribution of the particles. Such a model would sample the incoming particle's direction, position, and energy; therefore eliminating a full-scale simulation process.

Deng and co-workers [2] used the BEAMDP code to extract planar fluence, angular distribution, energy spectra and fractional sub-source contributions from EGS4/BEAM simulations of a Varian Clinac 2100C. Three photon subsources were modelled for particles originating from the target, primary collimator and flattening filter. The beam energies under consideration were 4, 6 and $15 \mathrm{MV}$. They could replicate 
dose distributions in patient models within $2 \%$ when compared between the source model and the original fully simulated phase space data [2].

Fix and co-workers [4] studied simple beam models generated for the GEANT4 code for 6 and $15 \mathrm{MV}$. Phase space data were collected for electron energies matching depth dose data. They set up four simple source models. In model 1, they used a single source with constant spatial fluence with a single energy spectrum. Model 2 varied this spatial fluence in the source retaining the energy spectrum as for model 1. In model 3 , they varied the energy spectrum keeping the spatial fluence the same as for model 1 . Finally, in model 4, they varied the spatial fluence and the energy spectra in the off-axis direction. Model 4 proved to be the most accurate over the range of field sizes studied [4].

Fippel et al. [9] developed a virtual energy fluence model using two Gaussian-shaped photon sub-sources and one uniform electron (contamination) source for the XVMC MC code. The photon sub-sources represented the bremsstrahlung target and the flattening filter. The parameters describing the Gaussian shape could be deduced from dose distributions in air for various square and rectangular fields with fixed monitor units [9].

Others improved the electron-contamination source model in a clinical photon beam for the EGS4-based BEAM and MCSIM codes. Its size depended on the field size with a predetermined energy spectrum from various accelerators. It could match clinical 6, 10 and $18 \mathrm{MV}$ photon beams within $2 \%$ or $2 \mathrm{~mm}$ [6].

Fix et al. [1] also studied photon source characteristics to construct a generalised three sub-source model representing photons originating from the target, primary collimator and flattening filter. They studied phase space data for 6 and 18 MV beams. They could achieve dose agreement for $99 \%$ of all voxels within $1 \%$ or $1 \mathrm{~mm}$ for the combined source model [1].

Fix et al. [1] also studied photon sub-source sensitivity to initial electron beam parameters for 6 and 18 MV. Again, they used three photon sub-sources and an additional primary electron sub-source, which allows for energy and radial distribution variation for electrons striking the linac target. They used phase space data for various electron energies striking the target as well as various radially spread distributions, which were dosimetrically compared between different data sets. The energy spread was found to have a small effect on the sub-source distributions while the mean energy and radial intensity changed the target sub-source distribution shape [10].

Sterpin et al. [11] studied the relationships between primary electron spot and focal size as well as the virtual source position in his MC model. They studied three electron energies between 3 and $18 \mathrm{MeV}$ with spot sizes ranging from 0 to 1.5 $\mathrm{mm}$ thickness for 0.15 and $1.0 \mathrm{~cm}$ thick tungsten targets. The virtual source position was determined through back projection of the photons from different phase space planes downstream. It was found the photon and electron spot size could be considered equal [11].

Smaller field size treatments involving stereotactic radiosurgery is also an area for MC source development such as works by Deng et al. [12] They built a dual source model for a 6 MV Cyberknife system. The EGS4 BEAM code was used for beam characterisation. Each source was circular for primary and scattered photons, respectively. No electron contamination was modelled. Dose data between phase space and the source model agreed within $2 \%$ or $2 \mathrm{~mm}$ for 6 to $60 \mathrm{~mm}$ diameter fields at 80 to $100 \mathrm{~cm}$ source-to-surface (SSD) fields [12].

Chaves and co-workers [13] used the MCNP4C to construct an eight-source model for a Siemens $6 \mathrm{MV}$ Megatron. The model could accurately calculate dose distributions in water for depth dose curves within $2.5 \%$ or $1 \mathrm{~mm}$ [13].

The aim of this study was to construct a hybrid photon source model for the EGSnrc-based MC code DOSXYZnrc for dose calculation to evaluate its effectiveness to model $6 \mathrm{MV}$ photon beams without the need for extra focal sub-sources. Beam particle fluence is modelled using suitable equations. The source is developed with the aid of an in-house code with a graphical user interface that allows for fluence adjustments in order to replicate clinical beam data. An additional planar electron contamination source was also included in the model. In this paper, the hybrid source model for the DOSXYZnrc MC code will be defined as the hybrid source model (HSM) and used throughout.

\section{Methods and Materials}

\section{Graphical user interface with beam modelling parameters}

A graphical user interface is used to adjust the parameters for the source to fit field profile data. It adjusts fluence intensities from the target to the exit plane by tweaking the fitting parameters of the beam modelling equations. Plots of the fluence can be displayed that act as a guide to adjust the exit fluence of the source model. The graphical user interface generates an output file that contains information regarding the energy spectrum and the fluence distribution. This file is then used as an input for the MC simulation.

The source consists of one circular photon source below the target. Its primary photon fluence is modelled with a Gaussian function [14].

$$
\phi_{t}=P \exp \left(\frac{-x^{2}}{\sigma^{2}}\right)
$$

$P$ is the amplitude of the primary fluence on the beam central axis $(\mathrm{CAX}) ; \sigma$ represents the full width at $50 \%$ intensity and $x$ is the radial distance from the CAX. The effect of the $\sigma$ value on the target fluence can be seen in Figure 1. 


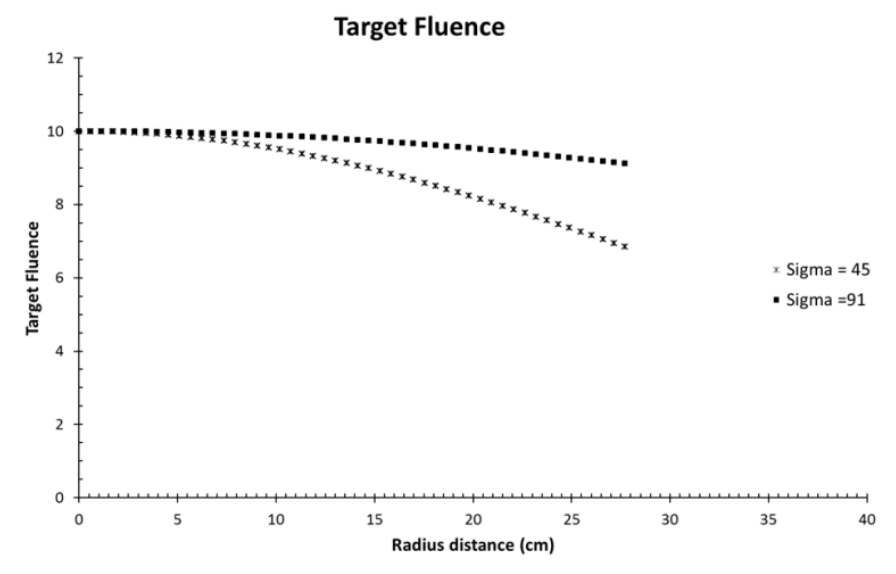

Figure 1. Effect of the $\sigma$ value on the target fluence

The photon fluence from the target passes through a conical shaped primary collimator. The primary collimator is modelled with error functions to correct the primary fluence for collimation and scattering. The transmitted photons under the collimators that add to the dose outside the field edges are modelled with an exponential function.

$$
\begin{aligned}
\phi_{p c}(r)= & \phi_{t} \operatorname{erf}\left(X_{3}, \sigma\right)+ \\
& 1\left[1-\operatorname{erf}\left(X_{3}, \sigma\right)\right] Z \exp \left(-\mu_{1}\left(r-X_{3}\right)\right)
\end{aligned}
$$

$\left[\operatorname{erf}\left(X_{3}, \sigma\right)\right]$ represents the fluence truncation effect of the collimators. The $\sigma$ is the penumbra width of the fluence collimated by the primary collimator. The value $\mu_{l}$ determines the rate of exponential reduction of the scattered primary fluence under the primary collimator as a function of $x$.

The bremsstrahlung energy spectrum is calculated using the modified Schiff method and is radially adjusted with an analytic function to model spectral softening due to the flattening filter [15,16-19].

It is a function of electron energy that will strike the target. The photon energy distribution is given by:

$$
\phi_{p}(E, r)=\frac{1}{E}\left[\begin{array}{l}
\left(1-\frac{E}{E_{0}}\right) \cdot(\ln \eta(E)-1)+ \\
\alpha(r)\left(\frac{E}{E_{0}}\right)^{2} \cdot(\ln \eta(E)-0.5)
\end{array}\right]
$$

$E_{0}$ and $E$ are the incident electron energy and the final electron energy respectively. The value for $\eta$ is obtained with the following equation:

$$
\eta(E)=\frac{1}{\sqrt{\left[\frac{0.511 E}{2 E_{0}\left(E_{0}-E\right)}\right]^{2}+\left[\frac{z_{w}^{1 / 3}}{111.0}\right]^{2}}}
$$

Eq. 4

The parameter $Z_{w}$ represents the tungsten target with an atomic number of 74. The value of 111.0 is Schiff's constant.

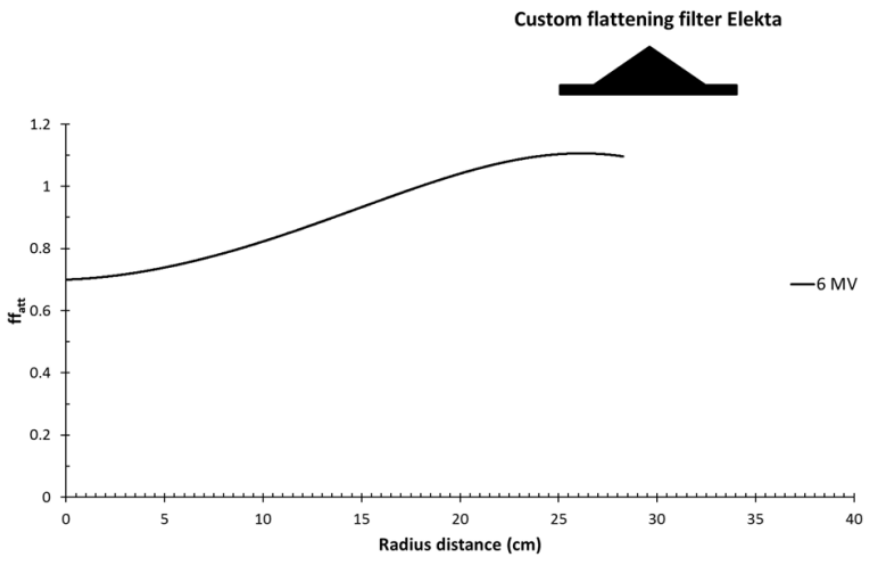

Figure 2. The attenuation caused by the flattening filter

The flattening filter models attenuation of the transmitted fluence from the source through an attenuation function, and can be seen in Figure 2:

$f f_{\text {att }}(x)=\exp \left(-\mu_{2} \cdot t\right)$

Eq. 5

Here $t$ is a polynomial describing the off-axis attenuation thickness.

The wedge was modelled in a similar way since its shape and atomic composition is known. The collimation of the jaws and multi-leaf collimators (MLC) was modelled with error functions as shown in Equation 6:

$\operatorname{erf}\left(X_{3}, \sigma\right)=\sqrt{1-\exp \left(-1.245 \times \frac{\left(x-X_{3}\right)^{2}}{\sigma^{2}}\right)}$

Eq. 6

Here $X_{3}$ denotes the $50 \%$ intensity of the penumbra and $\mathrm{x}$ is the off-axis position of the dose profile. The slope of the penumbra can be varied through parameter $\sigma$.

The mirror and monitor chamber was not modelled since they do not perturb the photon beam [14].

The direction of primary target photons is determined by a projection plane at an effective SSD. In this plane, an energy spectrum is associated at each point within a certain annulus to take off-axis beam softening into account due to the presence of the flattening filter $[8,20]$.

A planar electron contamination source is located just below the treatment head. Its energy spectrum was determined using a method that assumes that the percentage depth dose for a real clinical $6 \mathrm{MV}$ x-ray beam subtracted by a MC simulated pure photon beam of the same energy would yield the relative electron depth dose contribution to the clinical beam [21].

\section{Benchmarking water tank data acquisition}

For source commissioning, benchmarking clinical beam 3Ddose data must be available to set the parameters of the Gaussian circular photon source and error functions to conform to the dose profiles and depth dose of the water tank data. The 3D-dose data for other fields must be available to test the accuracy of the source model. Water tank scans of beam data 
were acquired to act as a benchmark against simulated MC data.

All the measurements for $6 \mathrm{MV}$ were taken with a Scanditronix Wellhöfer Blue Phantom system and OmniPro Accept software (version 6.5). A $0.01 \mathrm{~cm}^{3}$ PTW FREIBURG ${ }^{\circledR}$ PinPoint ionisation chamber was used for field measurements from $1 \times 1 \mathrm{~cm}^{2}$ to $5 \times 5 \mathrm{~cm}^{2}$ and a CC13 PTW FREIBURG ${ }^{\circledR}$ ionisation chamber for larger fields to $40 \times 40 \mathrm{~cm}^{2}$. At $100 \mathrm{~cm}$ SSD, dose profiles were measured at depths of 1.5, 5, 10, 20, and $30 \mathrm{~cm}$. Profiles in the inline- and crossline direction were made for field sizes from a $1 \times 1 \mathrm{~cm}^{2}$ field to a $40 \times 40 \mathrm{~cm}^{2}$ field. Wedged field profile data were acquired for a $10 \times 10$ $\mathrm{cm}^{2}, 20 \times 20 \mathrm{~cm}^{2}$ and a $30 \times 40 \mathrm{~cm}^{2}$ field. Offset profiles were acquired for $10 \times 10 \mathrm{~cm}^{2}, 15 \times 15 \mathrm{~cm}^{2}$ and $20 \times 20 \mathrm{~cm}^{2}$ fields in both the inline and crossline directions. Percentage depth dose curves were also measured.

\section{Monte Carlo simulations}

The beam characterisation model (Isource=4) is used that is incident from any direction. It consists of a polar coordinate system at the isocenter with a distance, dsource, to the center of the source plane. As seen in Figure 3, the origin of the plane is defined by theta and phi and the rotation of the source around its own plane is described by phicol.

Enough histories were simulated to reduce the variance below $1 \%$ in the clinical useful part of the beam. Photon transport was followed down to $10 \mathrm{keV}$ and electron transport was terminated at a total energy of $700 \mathrm{keV}$. The boundary crossing was set at 'EXACT' together with the PRESTA II electron-step algorithm. Spin effects were switched on with bound Compton scattering switched off. Atomic relaxations and Rayleigh scattering were not used.

The HSM dose data were scored at an SSD of $100 \mathrm{~cm}$ in a water phantom at a depth of $1 \mathrm{~cm}$. The resolution of the phantom $\left(60 \times 60 \times 60 \mathrm{~cm}^{3}\right)$ was $0.2 \mathrm{~cm}$ in the $x$ and $y$ directions and $0.5 \mathrm{~cm}$ in the $z$ direction.

\section{Verification of MLC aperture conformity}

In the source commissioning process, the HSM must also accurately replicate MLC apertures. Solid water Gafchromic film measurements for $6 \mathrm{MV}$ were compared with the source MLC apertures for an Elekta Synergy linear accelerator. A comparison was made between the $50 \%$ isodose lines at depths of 5 and $10 \mathrm{~cm}$. Irregular MLC shapes from Taskgroup 53 were planned on a $\mathrm{XiO}{ }^{\circledR} 4.62$ TPS for a phantom consisting of water. The treatment plan was transferred to the Elekta Synergy linac by using the Mosaiq software. GAFCHROMIC® EBT2 sheets (Lot No. A02011304), with dimensions $20.32 \times 25.4 \mathrm{~cm}^{2}$ were placed between water equivalent material that served as build-up and backscatter as in Figure 4.

The SSD was $100 \mathrm{~cm}$ and the film was irradiated at depths of 5 and $10 \mathrm{~cm}$, respectively. The monitor units (MUs) ranged between 327 and 358 for the different fields.

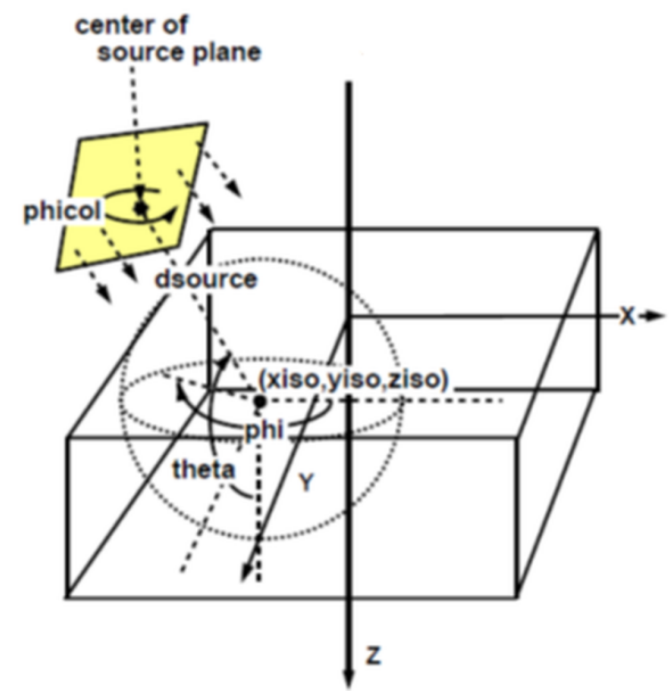

Figure 3. The configuration of the beam characterization model as seen in the DOSXYZnrc manual [8].

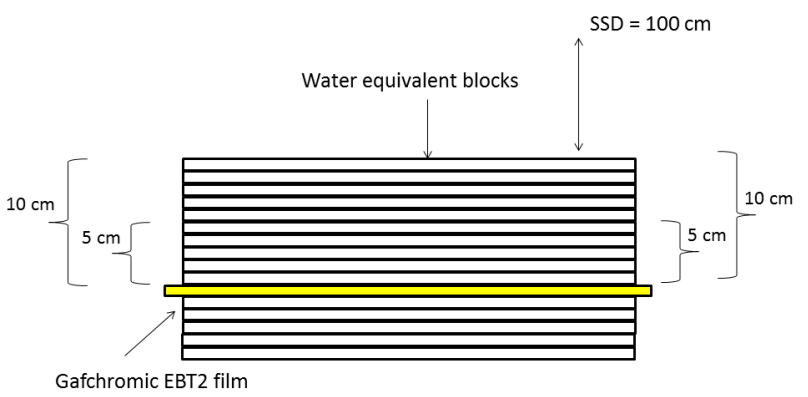

Figure 4. The set-up at the linac for the irradiation of the film inside the water equivalent phantom.

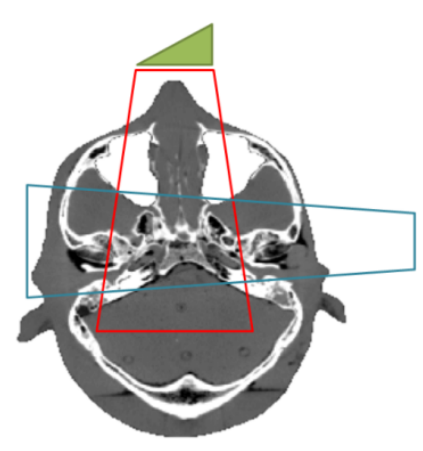

Figure 5. The plan set-up for the nasopharyngeal treatment.

\section{Simulation and verification of treatment plans}

The newly commissioned source was tested against Gafchromic film measurements in an anthropomorphic Rando phantom. Head-and-neck, prostate, and nasopharyngeal treatments were simulated.

One of the treatment plan consisted of two fields each with energy of $6 \mathrm{MV}$ as in Figure 5. A $7 \times 7 \mathrm{~cm}^{2}$ wedged field of 427 MUs with gantry angle of $0^{\circ}$ and a collimator angle of $90^{\circ}$ and a $5 \times 5 \mathrm{~cm}^{2}$ field of 256 MUs with a gantry angle of $90^{\circ}$ and a collimator angle of $0^{\circ}$ resulting in a uniform dose area. The nasopharyngeal treatment set-up can be seen in Figure 5. 
Films were scanned with an Epson Perfection V330 Photo scanner to produce uncompressed TIFF images. Before scanning, 24 hours were allowed for complete post irradiation polymerisation [20,22]. Afterwards, during scanning, film orientation was conserved with no image adjustment and colour corrections $[22,23]$. The resolution was 72 dpi in the 48bit colour depth mode. For dose comparison, a pixel-to-dose conversion curve was set up.

\section{Results}

\section{HSM parameters for the target and primary collimator}

The first step is to get the correct primary fluence as altered by the target, flattening filter and primary collimator. The largest field size, $40 \times 40 \mathrm{~cm}^{2}$, was used to determine the $\sigma$ value for the target fluence. In a least-square optimisation process, profile matching was calculated. The degree of agreement $(\alpha)$, which the sum of the square of differences between measured and calculated dose data, was minimised. Through adjustment of $\sigma$, the HSM dose was re-simulated and compared to the water tank data. A graph of $\alpha$ vs $\sigma$ revealed the optimal value for $\sigma$ in Equation 1. The result of an incorrect $\sigma$ value for Equation 1 can be seen in Figure 6.

The central part of the diagonal dose profile (at least $2.0 \mathrm{~cm}$ away from the penumbra) was not perturbed by the primary collimator, just the flattening filter.

From Figure 7, it can be seen that $\sigma=80$ would shape the primary fluence to yield the closest comparison between HSM and measured data by using the sum of the square of the dose difference between the two sets of data.

For the primary fluence for $\sigma=80$, the influence of the primary collimator is modelled by an error function, $\operatorname{erf}\left(x, \sigma_{l}\right)$, which truncates this fluence at the collimator edge (Figure 8). The value for sigma one, $\sigma_{1}$, describes the penumbra formed by the primary collimator.

The correct value for $\sigma_{1}$ was determined as 0.94 and remained fixed for the HSM. It was determined by the leastsquare fitment of the calculated and measured beam penumbra for the $40 \times 40 \mathrm{~cm}^{2}$ largest field on the diagonal profile. The same was done for the determination of the parameters for the MLC. The total fluence below the flattening filter can be seen in Figure 8.

\section{Hybrid source model parameters for MLC and jaws}

The effect of MLC and collimator truncation on the fluence is modelled by error functions, as in the case above for the primary collimator. For each field size there is an optimal $\sigma$ value that best describes the dose profile at the edge of the collimation device (penumbra). For the 'upper' jaws and the MLC, the optimal value was 0.4 , and for the 'lower' jaws, it was 0.07 , virtually constant over field sizes. The smaller value of $\sigma$ for the 'lower' jaw pair indicates a greater 'trimming' effect of the beam.

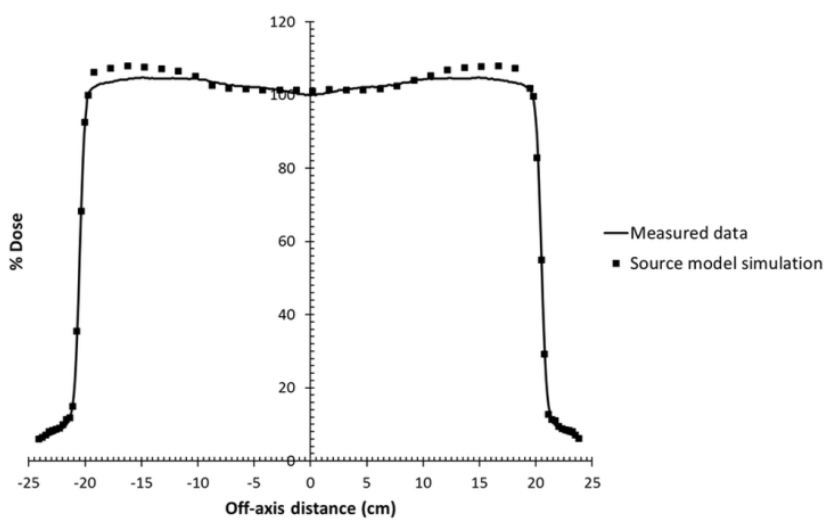

Figure 6. A comparison of dose profiles at $1 \mathrm{~cm}$ depth for a $40 \times$ $40 \mathrm{~cm}^{2}$ field size for an Elekta Synergy linear accelerator and simulated MC data. For this case, the dose is overestimated by the simulation and 'horns' are visible on the periphery of the field size.

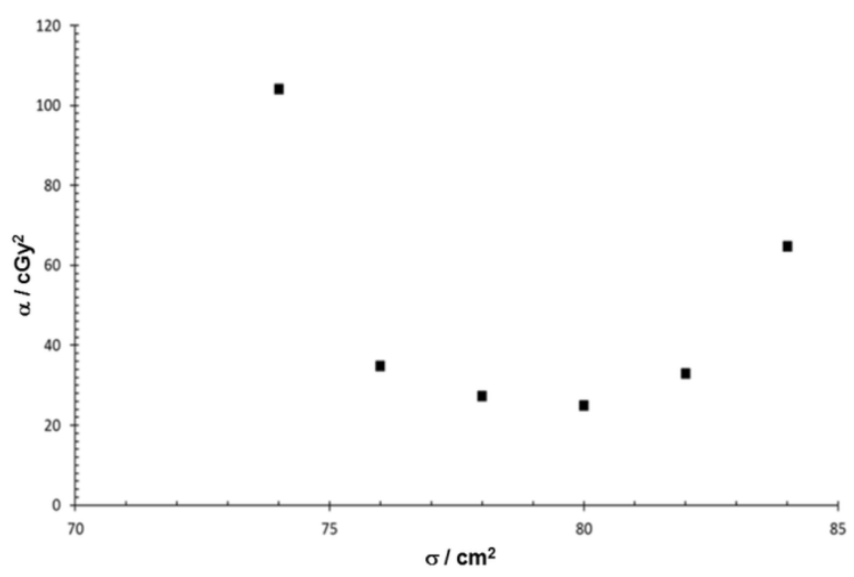

Figure 7. Values of $\alpha$ plotted against $\sigma$ where $\alpha$ is calculated as the sum of the square of the dose difference between measured and hybrid source model calculated diagonal dose profiles.

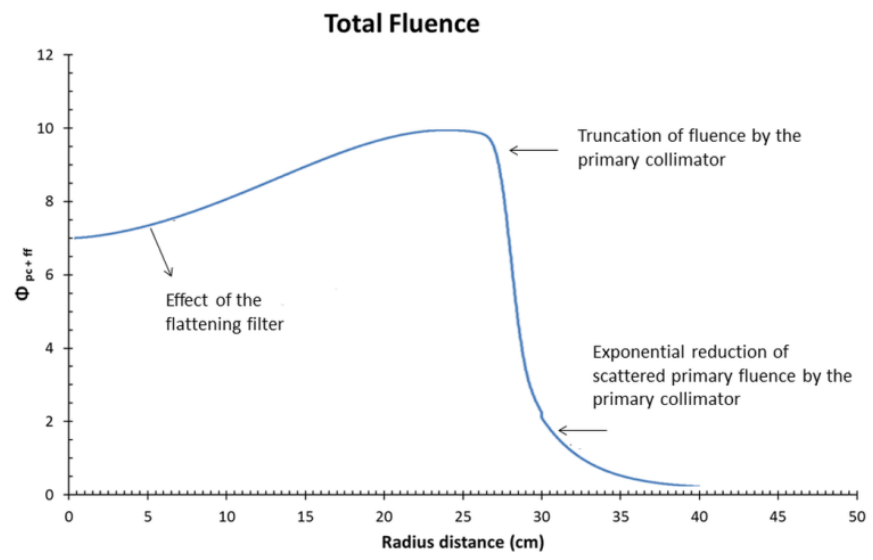

Figure 8. The total fluence below the flattening filter. The influence of the flattening filter on the primary fluence is shown. The primary fluence is truncated by the primary collimator and scatter is added by an exponential function. 
The effect of the $\sigma$ value on the jaws can be seen in Figure 9.

All the fields were firstly simulated with default parameters and compared with the measured data. The parameters were then altered until a good agreement was found between the simulated and measured data.

\section{Source commissioning: comparison between HSM and benchmark water tank data}

The dose profile data at depths of 1.5, 10, 20 and $30 \mathrm{~cm}$ are in good agreement (Figures 10-11). The same holds for half field dose profiles for fields larger than $10 \times 10 \mathrm{~cm}^{2}$ (Figure 12).

From the results, HSM fluence modelling allows for accurate dose profile replication compared with water tank measurements. The HSM was tested for offset and wedged fields (Figures 13, 14, and 15), and was found to be in good agreement with water tank data to within $1.5 \%$ or $1.5 \mathrm{~mm}$.

Good agreement was also found for the percentage depth dose (PDD) data for field sizes from $1 \times 1 \mathrm{~cm}^{2}$ to $5 \times 5 \mathrm{~cm}^{2}$ as seen in Figure 16.

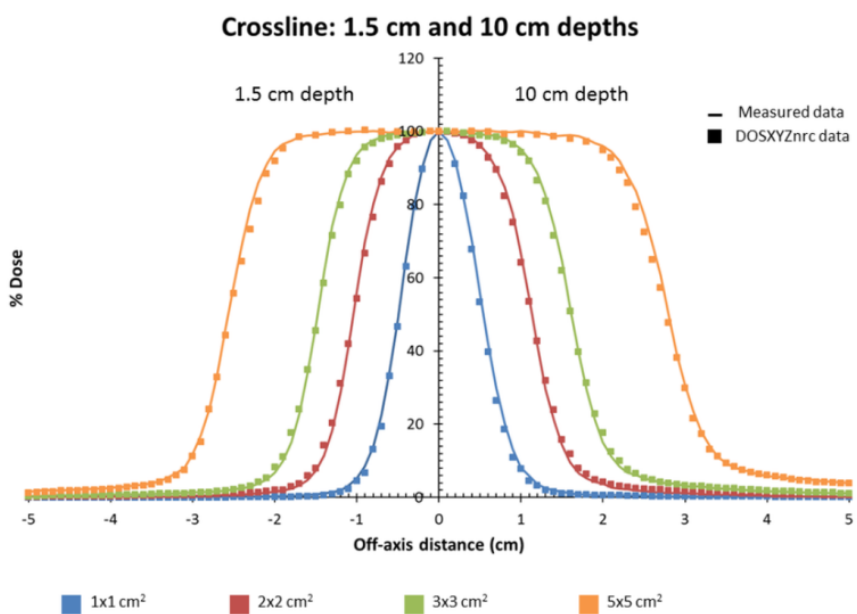

Figure 10. Dose profiles from water tank measurement and hybrid source model at 1.5 and $10 \mathrm{~cm}$ depths for fields ranging from $1 \times 1$ $\mathrm{cm}^{2}$ to $5 \times 5 \mathrm{~cm}^{2}$.

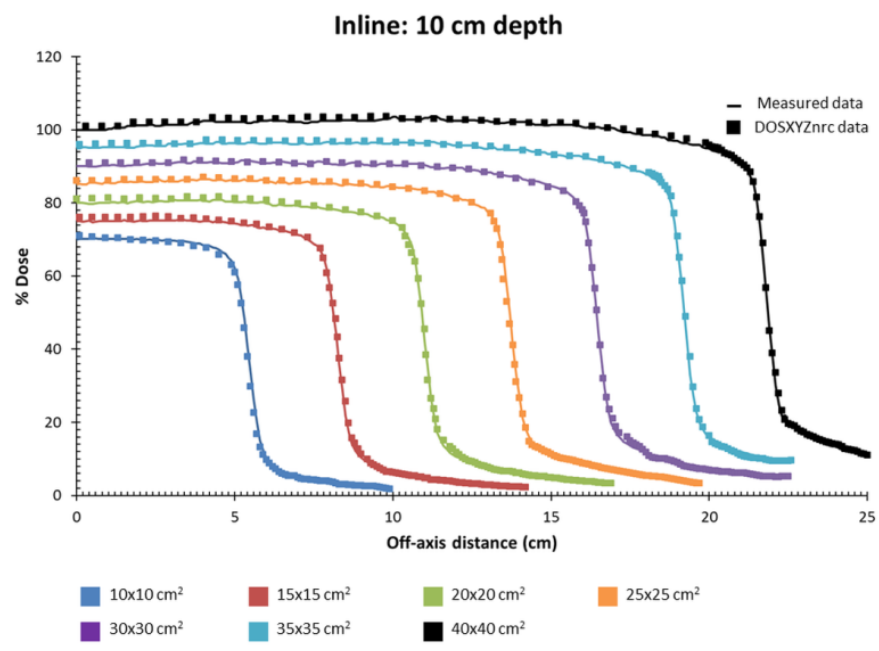

Figure 12. Half dose profiles from water tank measurement and hybrid source model at $10 \mathrm{~cm}$ depth for fields ranging from $10 \times 10$ $\mathrm{cm}^{2}$ to $40 \times 40 \mathrm{~cm}^{2}$.

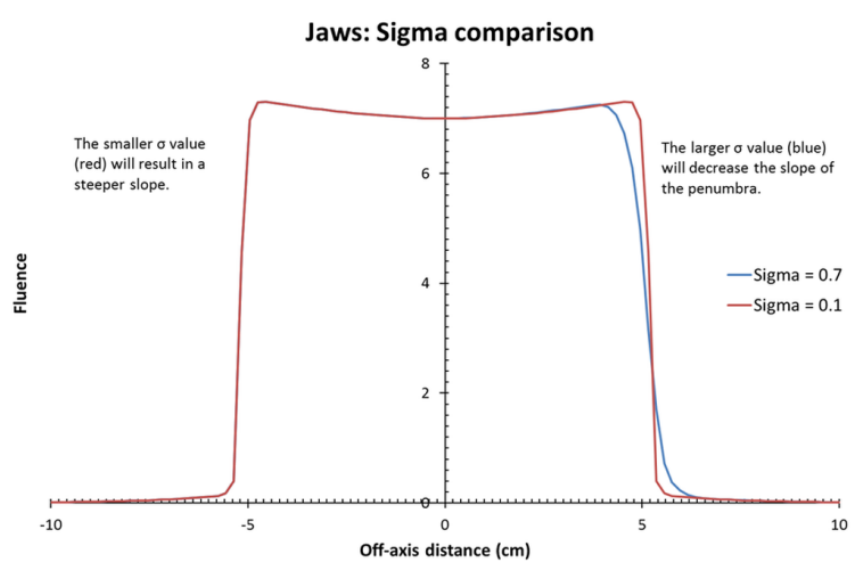

Figure 9. The effect of $\sigma$ on the penumbra width of the field.

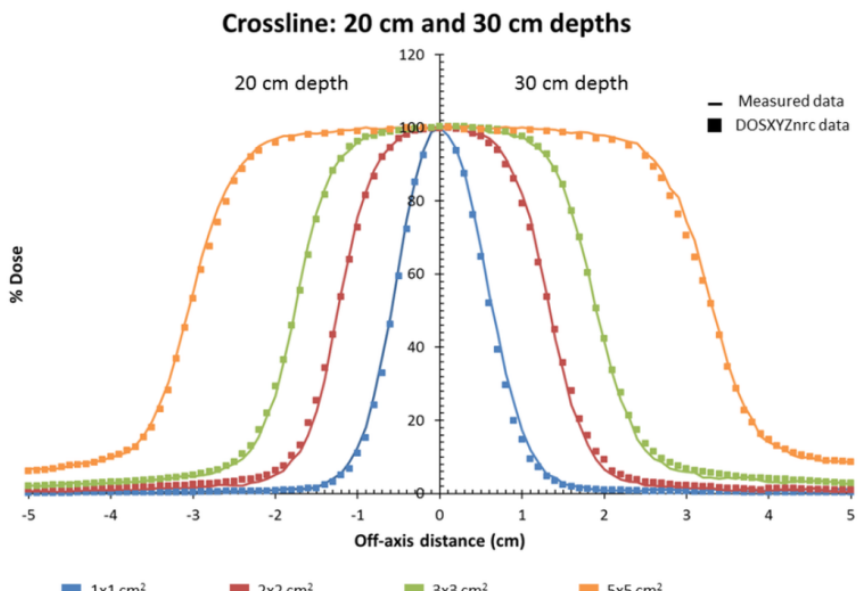

Figure 11. Dose profiles from water tank measurement and hybrid source model at 20 and $30 \mathrm{~cm}$ depths for fields ranging from $1 \times 1$ $\mathrm{cm}^{2}$ to $5 \times 5 \mathrm{~cm}^{2}$.

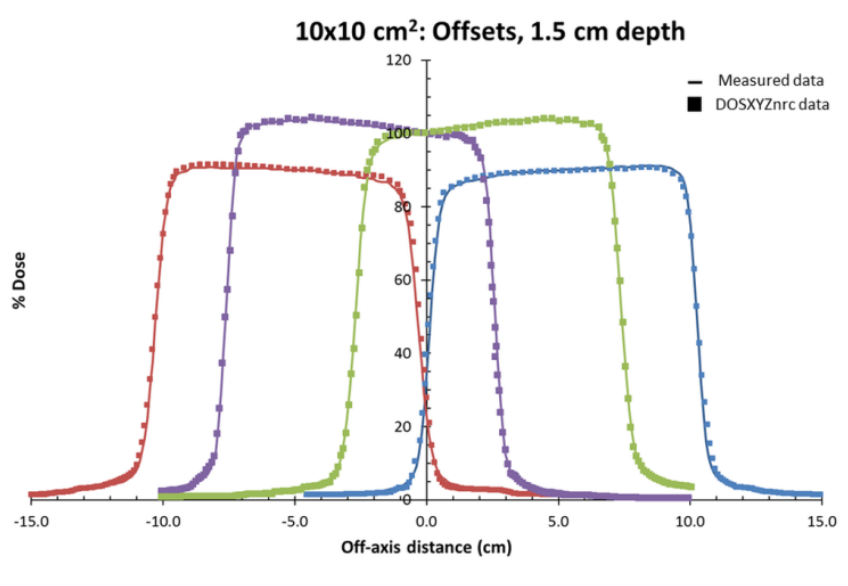

Figure 13. Dose profiles from water tank measurement and hybrid source model at $1.5 \mathrm{~cm}$ depth for a $10 \times 10 \mathrm{~cm}^{2}$ offset field. 


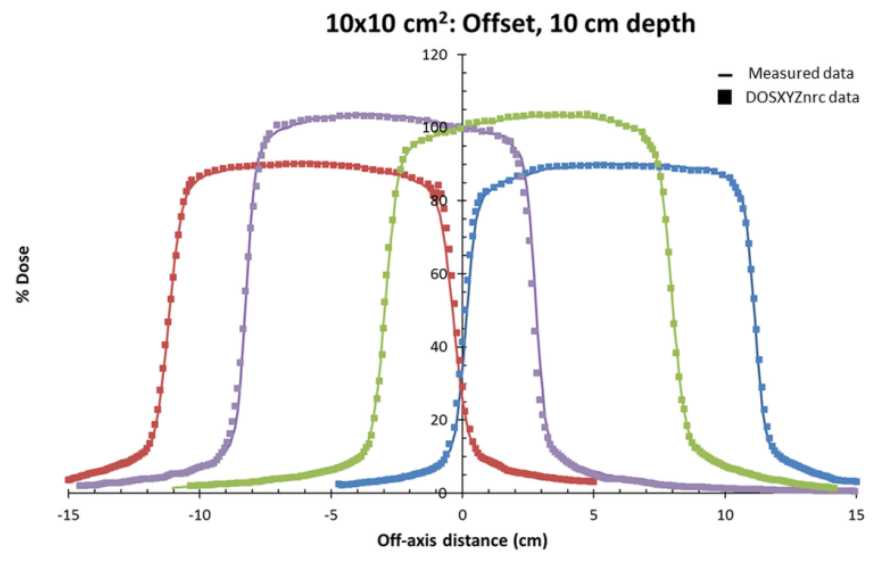

$10 \mathrm{~cm}$ offset, Inline

$10 \mathrm{~cm}$ offset, Crossline

$2.5 \mathrm{~cm}$ offset, Inline $2.5 \mathrm{~cm}$ offset, Crossline

Figure 14. Dose profiles from water tank measurement and hybrid source model at $10 \mathrm{~cm}$ depth for a $10 \times 10 \mathrm{~cm}^{2}$ offset field.

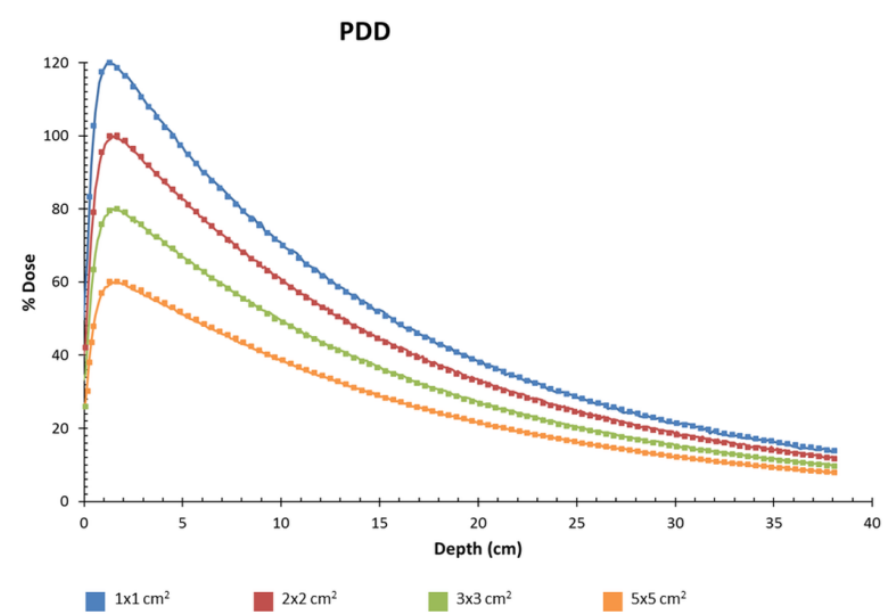

Figure 16. Percentage depth dose curves for field sizes $1 \times 1 \mathrm{~cm}^{2}$ to $5 \times 5 \mathrm{~cm}^{2}$

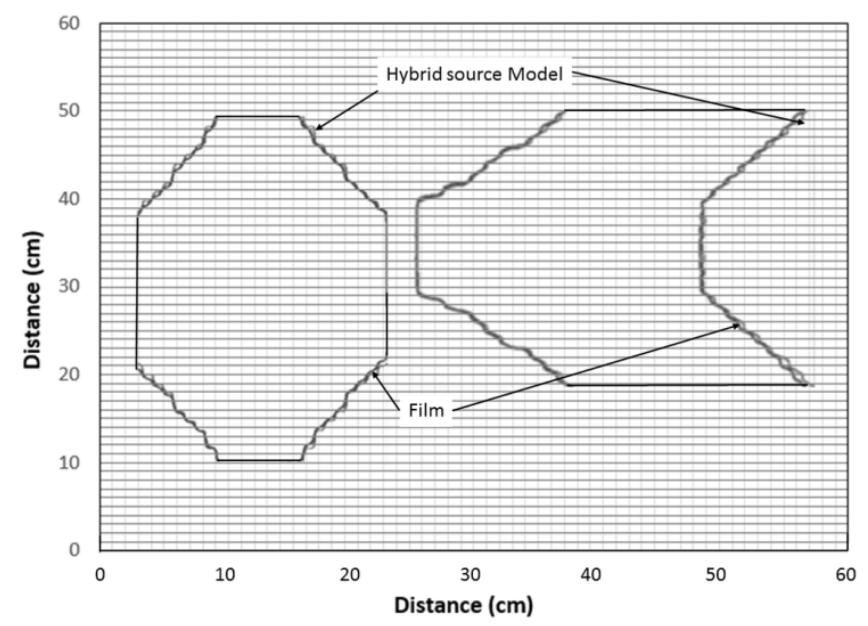

Figure 18. Multi-leaf collimators $50 \%$ isodose contour comparison between Gafchromic film (darker contour) and that calculated by the hybrid source model.

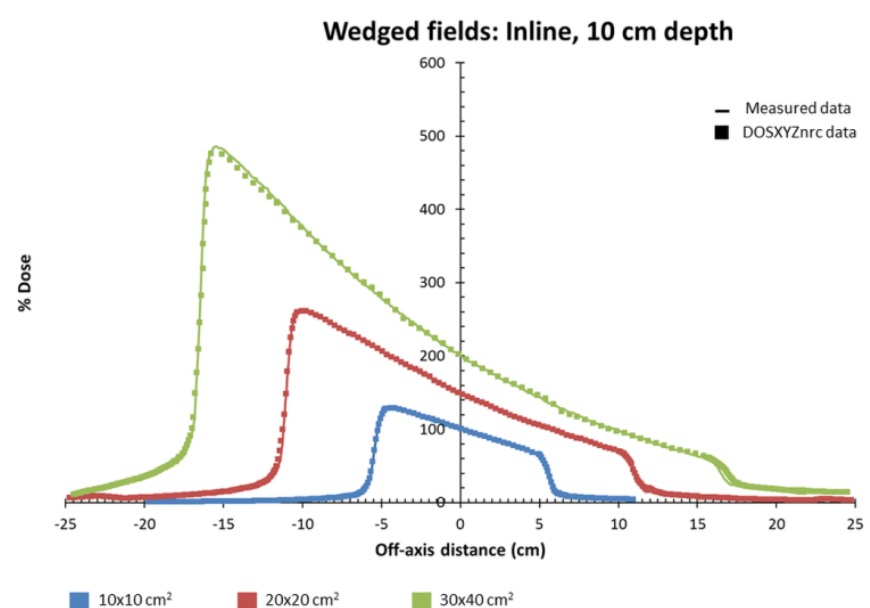

Figure 15. Relative dose profiles for measured and hybrid source model calculated dose profiles for wedge fields of $10 \times 10 \mathrm{~cm}^{2}, 20 \times$ $20 \mathrm{~cm}^{2}$, and $30 \times 40 \mathrm{~cm}^{2}$.

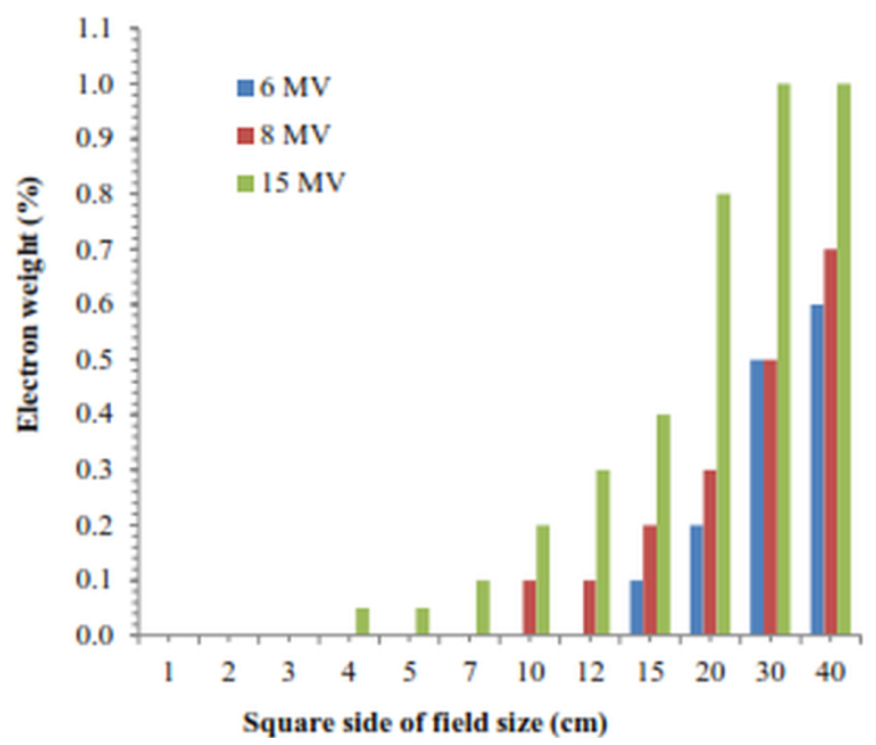

Figure 17. Relative weights for the planar electron contamination sub-source for the Elekta Synergy linear accelerator.

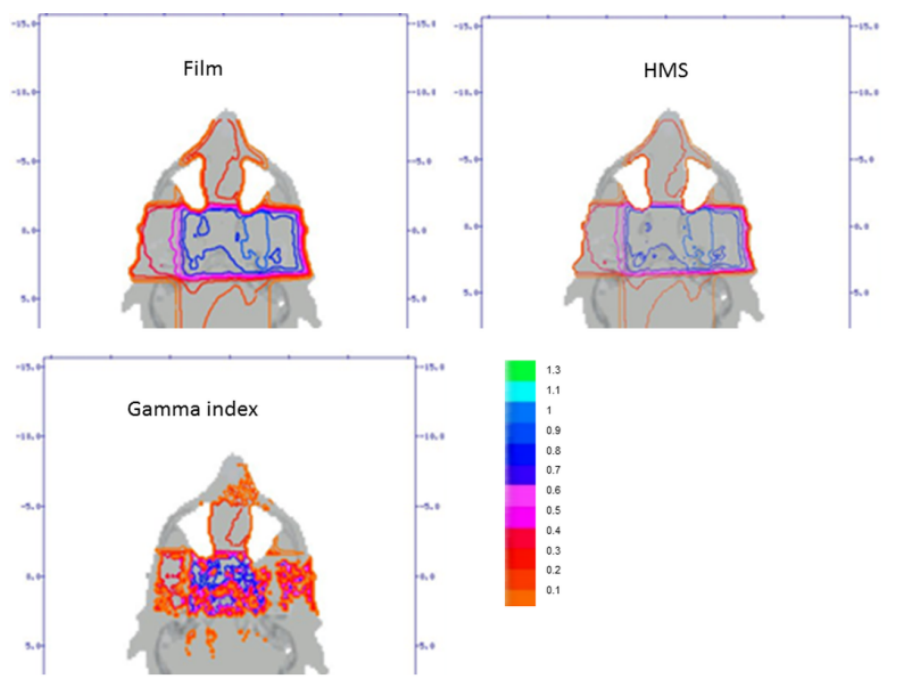

Figure 19. Nasopharyngeal cancer treatment; isodose lines for film and hybrid source model. The Gamma index evaluation is shown for a criterion of $3 \%$ or $3 \mathrm{~mm}$. 
The HSM consists of two sub-sources, one circular photon subsource and one planar sub-source for electron contamination. This is to enhance the surface dose of pure photon beams so that the real clinical beam can be replicated. The relative weight of the electron sub-source to create a combined dose that conforms to the relative dose of clinical beams was determined (Figure 17). The $6 \mathrm{MV}$ beam energy needs the least electron contamination that is only significant from fields exceeding $12 \times 12 \mathrm{~cm}^{2}$ whereas a $15 \mathrm{MV}$ beam needs to have electron contamination included for fields $>4 \times 4 \mathrm{~cm}^{2}$. The electron sub-source weight increases as the beam energy increases due to higher energy of secondary electrons needed to enhance the lower surface dose of the higher energy photons.

\section{Source commissioning: HSM conformity to MLC apertures}

The 50\% isodose line comparison for MLC contours simulated with the HSM and Gafchromic film measurements taken at 5 cm depth in a solid water RW3 phantom for an Elekta Synergy accelerator reveals that the worst discrepancy is within $4 \%$ or 4 mm while more than $95 \%$ of all points lie within $3 \%$ or $3 \mathrm{~mm}$ (Figure 18).

\section{Source commissioning: dose comparison between HSM and film measurements in a Rando phantom}

Gafchromic film and HSM dose data for nasopharyngeal treatment were compared by setting the Gamma criterion 3\% or $3 \mathrm{~mm}$, which was met for this complex dose calculation case involving soft tissue, bone structures and air cavities (Figure 19).

Uncertainties are attributed to Gafchromic film dosimetry, which contributes $1.5 \%$ in this study. The HSM variance is up to $1 \%$ and this enlarges dose error margins to larger than $2 \%$ or $2 \mathrm{~mm}$ when combined with film measurement.

\section{Discussion}

This study describes a HSM that contains a circular photonand electron contamination sub-source. The contribution of the latter is within $1 \%$ compared to the pure circular photon subsource weight. Off-axis differential spectral changes caused by the flattening filter were also included through a modified Shiff model.

The fluence truncation due to all collimators could be modelled with error functions. Collimator, flattening filter and wedge transmission was modelled with attenuation functions. Results indicated good replication (within $2 \%$ or $2 \mathrm{~mm}$ ) of calculated dose profiles and those measured in the water tank (Figures 10 to 16).

The HSM does not include additional sub-sources exclusively for secondary photon scatter modelling from collimator and jaw faces. As a result, output factors e.g. scatter factors and total scatter factors, cannot be calculated using this source. To ensure proper dose modelling, measured relative output scatter factors are used to scale each beam's dose contribution before they are combined to calculate the total dose. However, this does not limit the source model for its intended use.

For beam energies below $8 \mathrm{MV}$, electron contamination could be excluded in the model for fields $<12 \times 12 \mathrm{~cm}^{2}$. For higher beam energies at, say $15 \mathrm{MV}$, build-up modelling is compromised without an additional electron source. In some treatments, the dose close to the surface is sought and good agreement is a requirement against measured clinical data.

Another reason why a HSM approach for dose calculation was adopted is that radiation treatment fields are not always in the same plane (Z-slice). This causes difficulty for simple hand calculation for quality assurance purposes. This, in combination with high-contrast anatomical inhomogeneities, makes simple hand calculation challenging.

A deficiency of this HSM is that when treatment cases such as intensity-modulated radiation therapy (IMRT) are used, it would be better to include a full simulation of the accelerator to include all sources of machine scatter. This will take longer than just using a simple source model, but the total scattering will be accounted for. In this case, dose evaluation of IMRT plans will take much longer. However, this source model is suited for conformal therapy verification.

\section{Conclusion}

The HSM presented here consists of a single circular photon sub-source as well as a planar electron contamination subsource. The photon fluence was modelled by a Gaussian and the collimator scatter and truncation with error functions. The modified Shiff method was used to model the bremsstrahlung spectrum, which could also vary in the radial direction at offaxis locations. Determination of these function parameters could be done from regular beam data, including wedged fields. Asymmetrical fields could be modelled successfully. Examples of nasopharyngeal cancer treatment were used to show that the source model conforms to measurement within $3 \%$ or $3 \mathrm{~mm}$ on the linear accelerator for which it was implemented. This HSM is useful for dose calculation where regular or conformal fields are used. Care must be taken to ensure that the total scatter factor is known for each field shape. The HSM is also useful for evaluation of radiation dose, especially where the configuration of treatment fields makes simple hand calculation less accurate. For more advanced radiation treatment techniques, e.g. IMRT, a full MC simulation of the linac is recommended.

\section{Ethical approval}

All procedures performed in studies involving human participants were in accordance with the ethical standards of the institutional and/or national research committee and with the 1964 Helsinki declaration and its later amendments or comparable ethical standards. 


\section{Acknowledgements}

This research project was funded by the South African Medical Research Council with funds from National Treasury under its Economic Competitiveness and Support Package. This research and the publication thereof is the result of funding provided by the Medical Research Council of South Africa in terms of the MRC's Flagships Awards Project SAMRC-RFAUFSP-01-2013/HARD.
The authors thank Ms T Mulder, medical editor, School of Medicine, University of the Free State, for technical and editorial preparation of the manuscript.

\section{Conflict of interest}

The authors declare that they have no conflict of interest.

\section{References}

[1] Fix MK, Keall PJ, Dawson K, Siebers JV. Monte Carlo source model for photon beam radiotherapy: photon source characteristics. Med Phys. 2004;31(11):3106-3121.

[2] Deng J, Jiang SB, Kapur A, et al. Photon beam characterization and modelling for Monte Carlo treatment planning. Phys Med Biol. 2000;45(2):411-427.

[3] Chetty I, DeMarco JJ, Solberg TD. A virtual source model for Monte Carlo modelling of arbitrary intensity distributions. Med Phys. 2000;27(1):166-172.

[4] Fix MK, Keller H, Rüegsegger P, Born EJ. Simple beam models for Monte Carlo photon beam dose calculations in radiotherapy. Med Phys. 2000;27(12):2739-2747.

[5] Fix MK, Stampanoni M, Manser P, et al. A multiple source model for $6 \mathrm{MV}$ photon beam dose calculations using Monte Carlo. Phys Med Biol. 2001;46(5):1407-1427.

[6] Yang J, Li JS, Qin L, et al. Modelling of electron contamination in clinical photon beams for Monte Carlo dose calculation. Phys Med Biol. 2004;49(12):2657-2673.

[7] Keall PJ, Siebers JV, Libby B, Mohan R. Determining the incident electron fluence for Monte Carlo-based photon treatment planning using a standard measured data set. Med Phys. 2003;30(4):574-582.

[8] Walters B, Kawrakow I, Rogers DW. DOSXYZnrc Users Manual. NRCC Report PIRS-794revB. Ottawa, ON: National Research Council of Canada; 2009.

[9] Fippel M, Haryanto F, Dohm O, et al. A virtual photon energy fluence model for Monte Carlo dose calculation. Med Phys. 2003;30(3):301-311.

[10] Fix MK, Keall PJ, Siebers JV. Photon-beam subsource sensitivity to the initial electron-beam parameters. Med Phys. 2005;32(4):1164-1175.

[11] Sterpin E, Chen Y, Lu W, et al. On the relationships between electron spot size, focal spot size, and virtual source position in Monte Carlo simulations. Med Phys. 2011;38(3):1579-1586.

[12] Deng J, Guerrero T, Ma CM, Nath R. Modelling $6 \mathrm{MV}$ photon beams of a stereotactic radiosurgery system for Monte Carlo treatment planning. Phys Med Biol. 2004;49(9):1689-1704.

[13] Chaves A, Lopes MC, Alves CC, et al. A Monte Carlo multiple source model applied to radiosurgery narrow photon beams. Med Phys. 2004;31(8):2192-2204.

[14] Verhaegen F, Seuntjens J. Monte Carlo modelling of external radiotherapy photon beams. Phys Med Biol. 2003;48(21):R107-R164.

[15] Desobry GE, Boyer AL. Bremsstrahlung review: an analysis of the Schiff spectrum. Med Phys. 1991;18(3):497-505.

[16] Desobry GE, Boyer AL. An analytic calculation of the energy fluence spectrum of a linear accelerator. Med Phys. 1994;21(12):19431952.

[17] Baker CR, Peck KK. Reconstruction of $6 \mathrm{MV}$ photon spectra from measured transmission including maximum energy estimation. Phys Med Biol. 1997;42(11):2041-2051.

[18] Baker CR, Ama'ee B, Spyrou NM. Reconstruction of megavoltage photon spectra by attenuation analysis. Phys Med Biol. 1995;40(4):529-542.

[19] Partridge M. Reconstruction of megavoltage photon spectra from electronic portal imager derived transmission measurements. Phys Med Biol. 2000;45(10):N115-N131.

[20] Tailor RC, Tello VM, Schroy CB, et al. A generic off-axis energy correction for linac photon beam dosimetry. Med Phys. 1998;25(5):662-667.

[21] Smit C, du Plessis F. SU-E-T-238: deriving electron contamination spectra from pure and clinical photon beams. Med Phys. 2015;42(6):3387.

[22] Aldelaijan S, Mohammed H, Tomic N, et al. Radiochromic film dosimetry of HDR (192)Ir source radiation fields. Med Phys. 2011;38(11):6074-6083. 
[23] Butson MJ, Cheung T, Yu PK. Scanning orientation effects on Gafchromic EBT film dosimetry. Australas Phys Eng Sci Med. 2006;29(3):281-284 